Journal of ISMAC (2020)

Vol.02/ No. 02

Pages: $73-82$

http://irojournals.com/iroismac/

DOI: https://doi.org/10.36548/jismac.2020.2.001

\title{
Optimal Multipath Conveyance with Improved Survivability for WSN's In Challenging Location
}

\author{
Dr. Joy Iong Zong Chen, \\ Professor, Department of Electrical Engineering, \\ Da-Yeh University, \\ Taiwan. \\ Email id: jchen@mail.dyu.edu.tw
}

\begin{abstract}
The sensor networks with the capability of conveying information's through the wireless medium in many circumstances are affected by the inner energy and the exterior atmospheric changes as the sensors are employed in the areas that are beyond human reach and left uncared. Since routing procedure seems as the main source of energy utilization in these types of networks formulated by the sensors and as the prevailing routing procedures considers the parametric optimization from the network perception alone eluding the atmospheric influences over the network from the exterior sources, makes the sensors obtuse to the sudden unexpected changes in the atmosphere. So this leads to failure in conveyance of information's due to the failure in the routing and the fading of the path established. To manage such circumstance effectively the paper formulates a multi path conveyance with improved survivability for wireless-sensor-networks. The method takes into consideration the residual energy, depth and the dynamic changes in the atmosphere. The developed routing evades the information from passing over the hazardous area and forms a reliable conveyance by creating an atmosphere based on the monitoring competencies of the sensor network. The formulate method is experimentally evaluated using the network simulator-2 to acquire the vital enhancements in the life expectancy of the network framed and the delivery rate of the packets.
\end{abstract}

Keywords: Wireless sensor networks, Challenging Environment, Routing, Survivability, Life expectancy, Packet Delivery Ratio

\section{Introduction}

The swift and the rapid progress in the information sharing and the communication has afforded one to monitor and get useful insights form the surroundings and even form the places beyond human reach. This is made possible by the wireless -sensor-networks formed in an adhoc manner and capable of conveying information over air. These networks are constructed using multitudes of tiny sensor dispersed in the atmosphere with the capability of the sensing processing and communicating. The W-S-N are suitable for any types of atmosphere and are capable of self-configuring, healing and covering a broad scope of area. However in maximum circumstances W-S-Ns are required to track in the atmosphere where human reach is prohibited enabled by batteries, this causes the efficiency of the network to be easily influenced by the

ISSN: 2582-1369 (online) 
Journal of ISMAC (2020)

Vol.02/ No. 02

Pages: $73-82$

http://irojournals.com/iroismac/

DOI: https://doi.org/10.36548/jismac.2020.2.001

energy aspect and environmental causes; hence, the most important concern of WSNs is often guaranteed reliable data dispatching. Multipath conveyance protocols have now been used as the highly efficient method to ensure the data conveyance with heightened quality.

As the conveyance of data are liable of getting affected in the routing constructed with single trail as a result of failure caused in the node or link, conveyance in with multiple path seems to quiet convincing. Apart from less failures during delivery these multiple trails are competent enough to provide a proper balance in the network. So the multiple path conveyance has become a significant research concept. Most researchers developing the multiple trail discovery considers only the optimization of the network parameters such as energy, bandwidth, robustness etc., and does not take into consideration the dynamic atmospheric changes that affects, the sensing capability of the network. The sudden changes happening in the atmosphere turns the sensors obtuse, causing, failure in the conveyance and damages in the network. For instance an extreme temperature would either cause the sensor to breakdown or even burn the nodes involved in sensing, whereas an extremely high humidity would cause the short circuits in the sensors affecting the quality in the link, and the inference caused due to EM also would end up with the loss in the data rate. So it become essential to form a trail that does not cross the hazardous area, the trail discovered has to be responsive of the surrounding to avoid the data losses and enhance the delivery rate. So the method formulated in paper also considers the environmental changes along with the residual energy and the depth to develop a multi trail conveyance with heightened survivability, life expectancy and delivery rate of packets. The proposed work mainly scopes in providing an

1. Reliable Routing in the challenging atmospheric conditions, according to the data obtained from the sensors.

2. Responsive trails with the capability to avoid the hazardous area,

3. Enhanced latency, energy usage and a better load balance with increased life expectancy.

The paper constructing an atmospheric responsive trail with the enhanced life expectancy and data delivery is planned with the related works in section 2, proposed multipath conveyance that is atmospheric responsive in section.3, Experimental analysis in section 4 and conclusion in section 5

\section{Related Works}

The idea motivating the multipath conveyance started since 1990 in order to improve the balance in the load and achieve tolerance against errors in the wireless adhoc networks. Nowadays the same is utilized in W-S-N to enhance the reliability of the trails of the energy usage of the networks. The research the

ISSN: 2582-1369 (online) 
Journal of ISMAC (2020)

Vol.02/ No. 02

Pages: $73-82$

http://irojournals.com/iroismac/

DOI: https://doi.org/10.36548/jismac.2020.2.001

methodology followed by the authors to obtain a multiple trails to convey the information through the W$\mathrm{S}-\mathrm{N}$ is described in the related work section Lou, Wenjing et al [1] has put forth a "first node disjoint routing protocol" by using the spanning tree and a simple flooding strategy for the "wireless sensor networks"

Younggoo Kwon et al [2] performed the modified process of the method developed by Lou, engaging a mechanism with secret sharing setting the verge level in the HSpread,, to reestablish the connections and restore the messages in the sink even on the occurrence of failures in certain paths, this method focused on avoiding the link failures and eluded the energy optimization.

So the author $\mathrm{Li}$, et al [3] in his paper performed an. "Efficient multi-path protocol for wireless sensor networks." Capable of providing a delay tolerant, improved throughput and energy efficient model that used almost all the resources available in the network. Raj, Jennifer S. et al [4] conducted a "QoS optimization of energy efficient routing in IoT wireless sensor networks" employing the fuzzy and the CNN.

Wang, et al [5] presented the accident responsive, energy optimized trailing for the WSN applying the segregation process across the source and the destination and by establishing independent path, and this was performed to lower the mutual interference in the trail.

Kumar et al [6] performed the node disjoint routing based on the geographic details of the sensors. The nearby nodes with the minimum angle to the sink were sort out and used in the process. The method the presented a better latency.

Jannu, Srikanth et al [7] conducted a. "A grid based clustering and routing algorithm for solving hot spot problem in wireless sensor networks." Smys, S., and Robert Bestak et al [8] discussed the "Introduction to the Special Issue on Inventive Network Structures for Next Generation Wireless Personal Systems." Kominami, et al [9] has performed the "Controlled and self-organized routing for large-scale wireless sensor networks."

Smys, S. et al [10] put forth an "Energy-Aware Security Routing Protocol For WSN in Big-Data Applications." Pham, Congduc et al [11] Communication performances of IEEE 802.15. 4 wireless sensor motes for data-intensive applications: A comparison of WaspMote, Arduino MEGA, TelosB, MicaZ and iMote2 for image surveillance."

ISSN: 2582-1369 (online) 
Journal of ISMAC (2020)

Vol.02/ No. 02

Pages: $73-82$

http://irojournals.com/iroismac/

DOI: https://doi.org/10.36548/jismac.2020.2.001

Wang, et al [12] performed the. "PWDGR: pair-wise directional geographical routing based on wireless sensor network." Yi et al [13]. "Security-aware ad hoc routing for wireless networks." Sivaganesan, D et al [14] put forth the "Efficient Routing Protocol with Collision Avoidance in Vehicular Networks."

\section{Proposed Work}

The proposed work in order to discover an atmospheric alert routing to avoid crossing the hazardous area utilizes the potential field method put forth by the Khatib, and Oussama et al [15] the authors developed the potential field to handle the movement of the robots, between the source to destination by using the resultant force of repulsion the attraction, the robots were able to avoid the obstacle and move forward towards the destination correspondingly with the application of repulsion and attraction force.

In the proposed model the information packets sent by the source to the destination in similar way as the robots are initiated from source to the destination, assigning the motes in the path as the signs for direction of traversing. So the method formulated used the potential field in [15] in the WSN data conveyance from source to destination the potential field with the resultant force of attraction and repulsion were engaged to avoid the data loss caused due to the hazardous environment. The destination acts as the attracting force, the sensor in the path behave like the direction boards and the dangerous zones degree are proportional to the repulsion force that is indirectly proportional to the remaining energy. With this the proposed method develops an adjustable multiple trail to avoid the hazard areas and identify the robust and secure path. The flow diagram in fig. 1 below shows the stages in the trail discovery and maintenance of the proposed method.

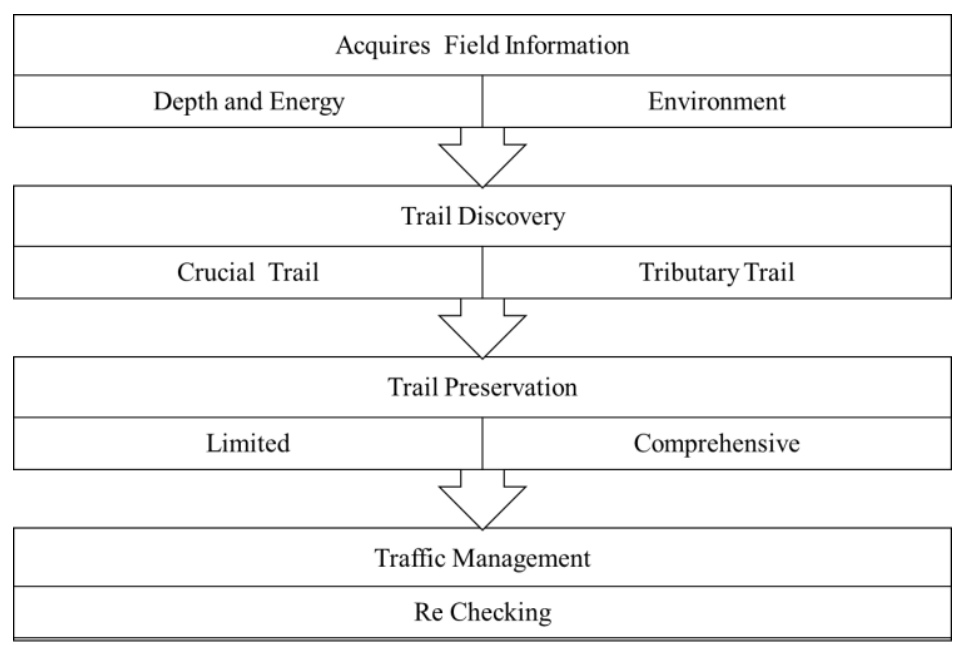

Figure.1 Flow Diagram

ISSN: 2582-1369 (online) 
Journal of ISMAC (2020)

Vol.02/ No. 02

Pages: $73-82$

http://irojournals.com/iroismac/

DOI: https://doi.org/10.36548/jismac.2020.2.001

The tiny sensing device in order to make the routing decision acquires the field information such as the depth (D), remaining energy (RE) of the motes and the environment (ENV) details. To make the sensors aware of the environment, in the work carried out it is assumed that the sensor nodes are capable enough to draw the details of the environment. This made possible by the message interchange that takes place among the nearby nodes, each node affords to get the details of the atmosphere around it and reserves the information in the table used for storing the routing particulars.

Once the information about the environment is gathered the trail to the destination is sorted out, in two strides, the first stride gathers the information of the field and the second strides identifies the paths, first the crucial trial and then the tributary trail. The algorithm below in figure. 2 states the path discovery of the crucial and the tributary trail.

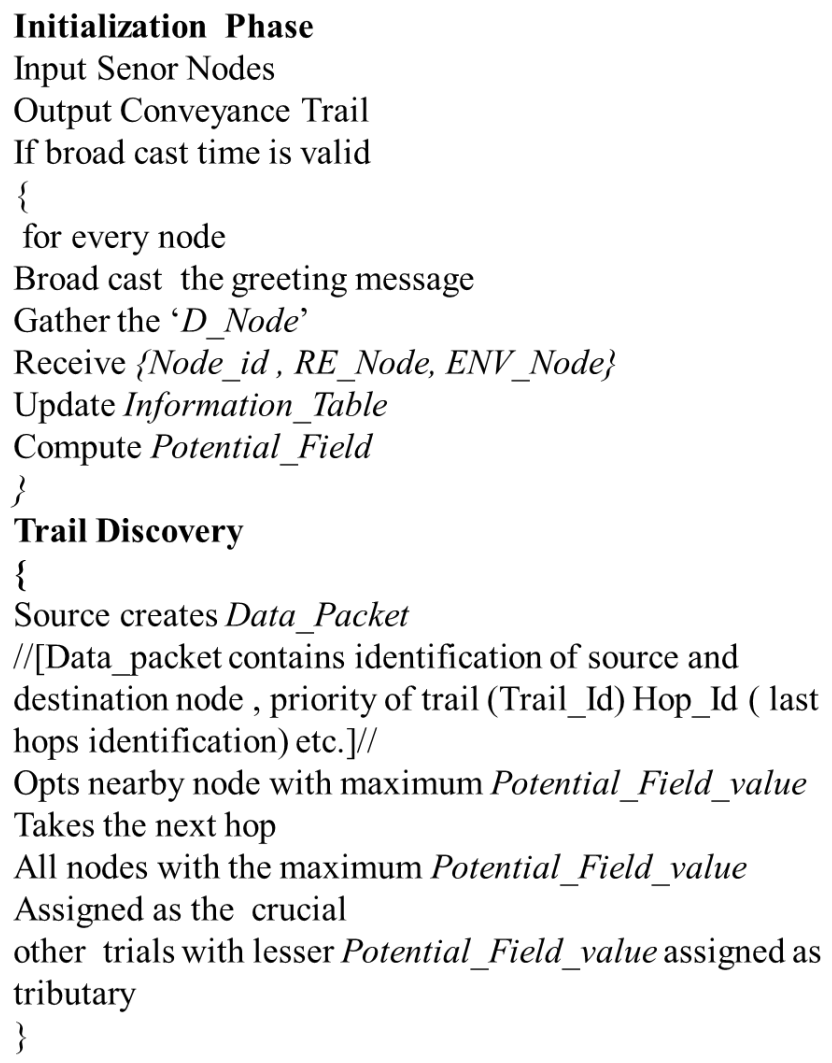

Figure.2 Trail Discovery

ISSN: 2582-1369 (online) 
Journal of ISMAC (2020)

Vol.02/ No. 02

Pages: $73-82$

http://irojournals.com/iroismac/

DOI: https://doi.org/10.36548/jismac.2020.2.001

Once the trail is discovered the trails are maintained by performing an automatic update, sending frequently greeting message to the nearby nodes on the receiving the greeting messages the nearby nodes sends the current status of the nodes regarding the "RE", "D" and the "ENV" this is termed as the limited update, the limited update is performed among the adjacent nodes to preserve the trails, further the destination node performs a comprehensive update by flooding the updated " $D$ " to the entire network at a regular instance. The conveyance is managed with proper traffic management by allocating the crucial trails for major of the transmission and assigns the secondary only when the all the crucial paths are in use. The proposed methodology follows a rechecking to avoid dropping of packets and instead resending back to the previous hop by tracing the previous hop_id form the table holding the routing information and later identifies the alternative node to continue with the transmission process without stopping the conveyance. Figure. 3 below depicts the rechecking process.

\section{Performance Evaluation}

The formulated method following a proactive method of routing by considering the environmental information's to elude the data loss because of the sudden environment changes, is simulated using the NS2 with the following parameter used for the simulation. The table. 1 below shows the simulation parameters.

\begin{tabular}{|l|l|}
\hline \multicolumn{1}{|c|}{ Parameters } & \multicolumn{1}{c|}{ Values } \\
\hline Sensor nodes & 500 \\
\hline Simulation Time & 10000 Seconds \\
\hline Simulation area & $40 \mathrm{mx} 40 \mathrm{~m}$ \\
\hline Initial Energy & 1 joule/sensor node \\
\hline Packet Bytes & 125 bytes \\
\hline Transmission Range & $50 \mathrm{~m}$ \\
\hline $\begin{array}{l}\text { Energy Consumption } \\
\text { Model }\end{array}$ & First -order radio model \\
\hline Mobility of Nodes & Stationary \\
\hline
\end{tabular}

Table.1 Simulation Parameters

ISSN: 2582-1369 (online) 
Journal of ISMAC (2020)

Vol.02/ No. 02

Pages: $73-82$

http://irojournals.com/iroismac/

DOI: https://doi.org/10.36548/jismac.2020.2.001

The method is simulated on the basis of delivery rate and the network life expectancy and the end to end delay. The figure. 3 below is the delivery rate of the packet achieved in the proposed model and the over different environment circumstance such as the forest fire, storms, etc. this is compared with the existing method, that follows a proactive multiple path routing without the awareness of the environment (EMPR) and the AODV

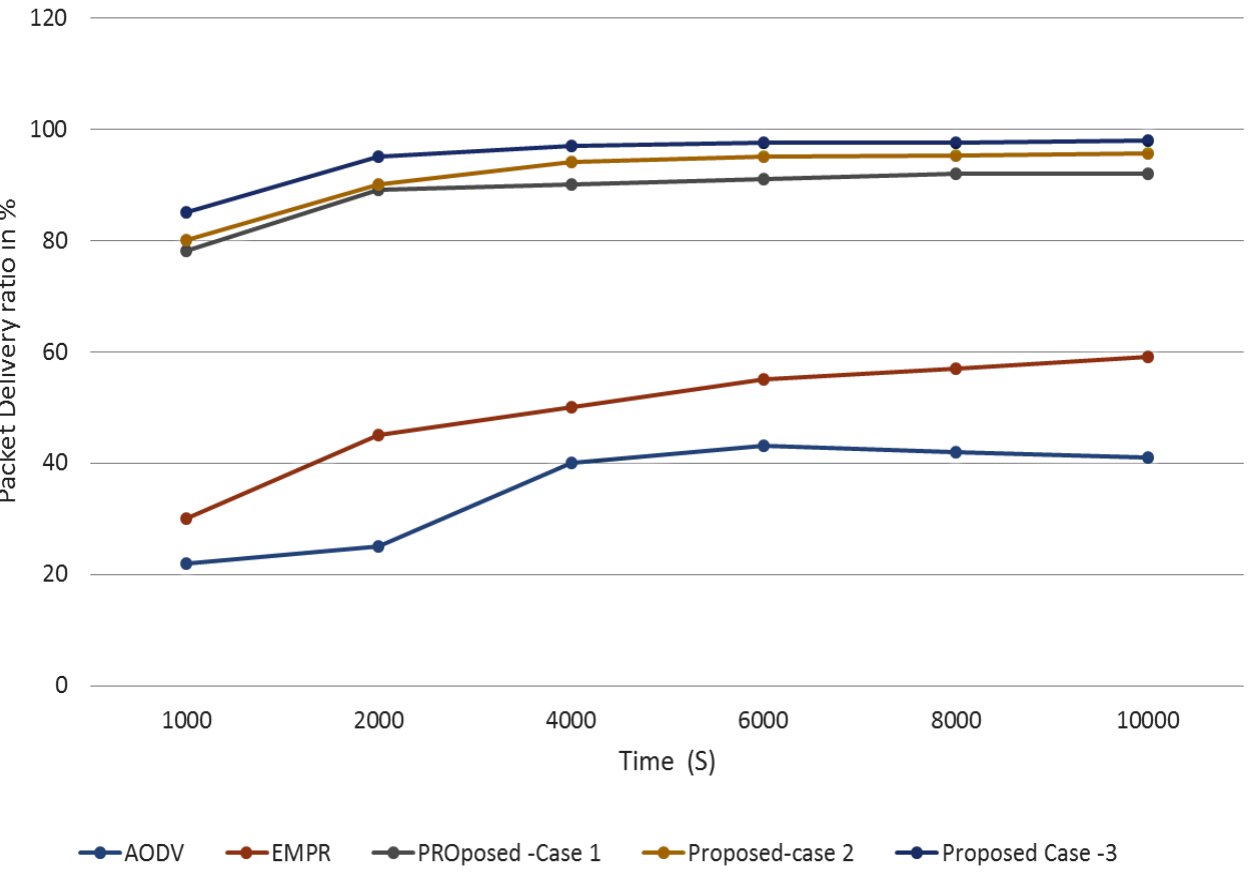

Figure.3 Delivery Rate of Packets

The packet delivery is described "as the ratio of the number of the packets successfully received by the destination W.R.T the average number of packets sent by the nodes employed for sensing". The figure.4 below shows the life expectancy of the network, "it is defined as the duration during which the half of the amount sensor nodes turn into failure"

ISSN: 2582-1369 (online) 
Journal of ISMAC (2020)

Vol.02/ No. 02

Pages: $73-82$

http://irojournals.com/iroismac/

DOI: https://doi.org/10.36548/jismac.2020.2.001

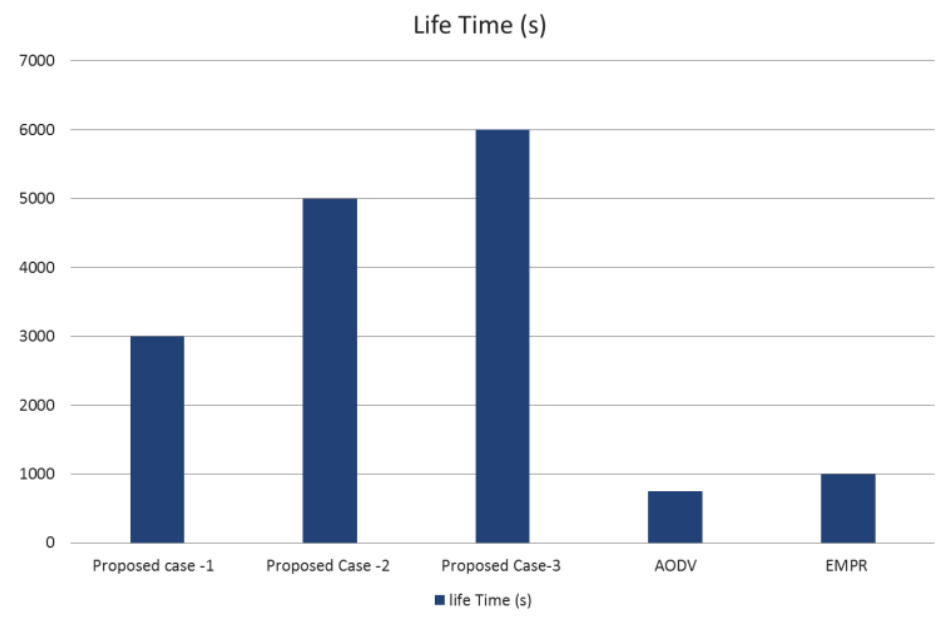

Figure.4 Life Expectancy

The results observed shows that the proposed method is very much better compared to the existing method that follows a proactive multiple path routing without the awareness of the environment and the AODV.

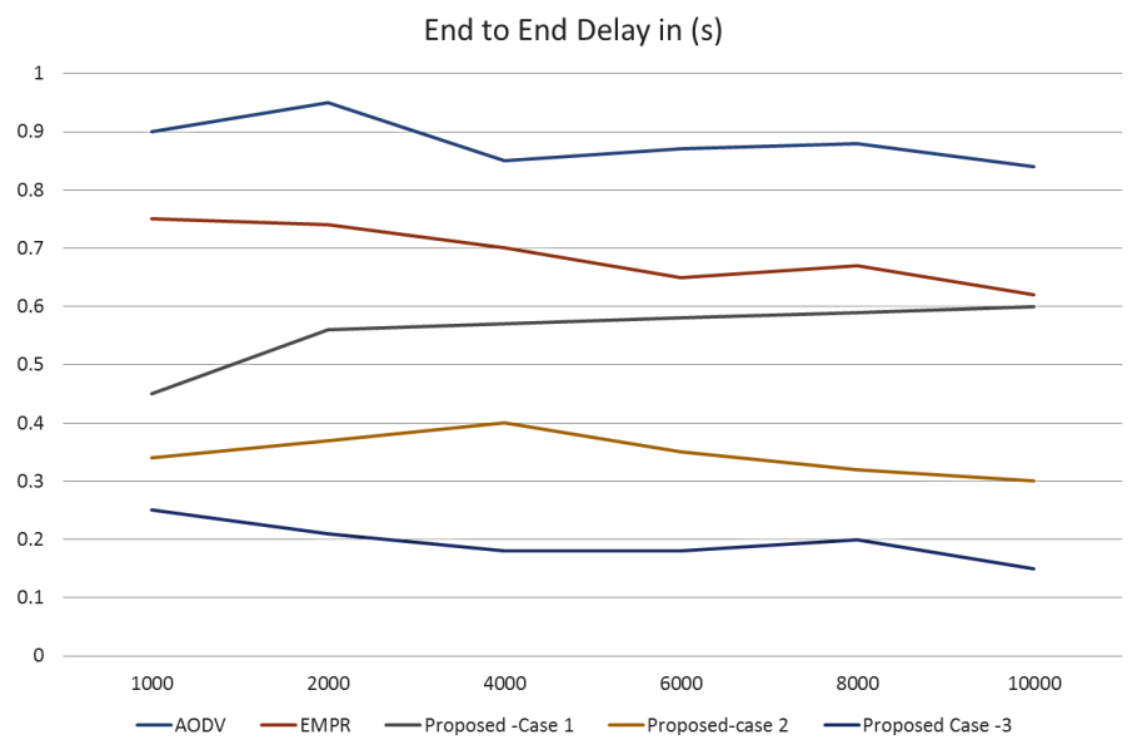

Figure.5 End to End Delay

ISSN: 2582-1369 (online) 
Journal of ISMAC (2020)

Vol.02/ No. 02

Pages: $73-82$

http://irojournals.com/iroismac/

DOI: https://doi.org/10.36548/jismac.2020.2.001

"The total time taken by the packets to reach the destination, including the time of computation, queuing, conveyance and propagation etc." is referred as the delay caused in the transmission. The delay measured for the conveyance on different cases applying the proposed method is compared with the existing method that follows a proactive multiple path routing without the awareness of the environment and the AODV and proved to have an improved performance as shown in figure. 5 .

\section{Conclusion}

The paper formulates a method to sort out a safe trail for the conveyance of data without affecting the information in the data. This is made possible by developing a trail enriched with the environment particulars and the potential field value, to avoid the path under the hazard zone and reach the destination safely without the data loss. The method put forth is simulated using the NS-2 on the basis of the delivery rate of packets, life expectancy and the end to end delay and is compared with the other existing methods such as the EMPR and the AODV and proved to have better performance compared to the other two under different circumstance.

\section{References}

[1] Lou, Wenjing. "An efficient N-to-1 multipath routing protocol in wireless sensor networks." In IEEE International Conference on Mobile Adhoc and Sensor Systems Conference, 2005., pp. 8-pp. IEEE, 2005.

[2] Lou, Wenjing, and Younggoo Kwon. "H-SPREAD: a hybrid multipath scheme for secure and reliable data collection in wireless sensor networks." IEEE Transactions on Vehicular Technology 55, no. 4 (2006): 1320-1330.

[3] Li, Shuang, Raghu Kisore Neelisetti, Cong Liu, and Alvin Lim. "Efficient multi-path protocol for wireless sensor networks." International Journal of Wireless and Mobile Networks 2, no. 1 (2010): 110-130.

[4] Raj, Jennifer S. "QoS optimization of energy efficient routing in IoT wireless sensor networks." Journal of ISMAC 1, no. 01 (2019): 12-23.

[5] Wang, Zijian, Eyuphan Bulut, and Boleslaw K. Szymanski. "Energy efficient collision aware multipath routing for wireless sensor networks." In 2009 IEEE International Conference on Communications, pp. 1-5. IEEE, 2009.

[6] Kumar, Alok, and Shirshu Varma. "Geographic node-disjoint path routing for wireless sensor networks." IEEE Sensors journal 10, no. 6 (2010): 1138-1139.

ISSN: 2582-1369 (online) 
Journal of ISMAC (2020)

Vol.02/ No. 02

Pages: $73-82$

http://irojournals.com/iroismac/

DOI: https://doi.org/10.36548/jismac.2020.2.001

[7] Jannu, Srikanth, and Prasanta K. Jana. "A grid based clustering and routing algorithm for solving hot spot problem in wireless sensor networks." Wireless Networks 22, no. 6 (2016): 1901-1916.

[8] Smys, S., and Robert Bestak. "Introduction to the Special Issue on Inventive Network Structures for Next Generation Wireless Personal Systems." Wireless Personal Communications 90, no. 2 (2016): 421-422.

[9] Kominami, Daichi, Masashi Sugano, Masayuki Murata, and Takaaki Hatauchi. "Controlled and self-organized routing for large-scale wireless sensor networks." ACM Transactions on Sensor Networks (TOSN) 10, no. 1 (2013): 1-27.

[10] Smys, S. "Energy-Aware Security Routing Protocol For WSN in Big-Data Applications." Journal of ISMAC 1, no. 01 (2019): 38-55.

[11] Pham, Congduc. "Communication performances of IEEE 802.15. 4 wireless sensor motes for data-intensive applications: A comparison of WaspMote, Arduino MEGA, TelosB, MicaZ and iMote2 for image surveillance." Journal of Network and Computer Applications 46 (2014): 4859.

[12] Wang, Junfeng, Yin Zhang, Jialun Wang, Yujun Ma, and Min Chen. "PWDGR: pair-wise directional geographical routing based on wireless sensor network." IEEE internet of things journal 2, no. 1 (2014): 14-22.

[13] Yi, Seung, Prasad Naldurg, and Robin Kravets. "Security-aware ad hoc routing for wireless networks." In Proceedings of the 2nd ACM international symposium on Mobile ad hoc networking \& computing, pp. 299-302. 2001.

[14] Sivaganesan, D. "Efficient Routing Protocol with Collision Avoidance in Vehicular Networks." Journal of Ubiquitous Computing and Communication Technologies (UCCT) 1, no. 02 (2019): 76-86.

[15] Khatib, Oussama. "Real-time obstacle avoidance for manipulators and mobile robots." In Autonomous robot vehicles, pp. 396-404. Springer, New York, NY, 1986.

Biography: Dr. Joy Iong-Zong Chen is currently a full professor of Department of Electrical Engineering Dayeh University at Changhua Taiwan. Prior to joining the Dayeh University, he worked at the Control Data Company (Taiwan) as a technical manager since Sep. 1985 to Sep. 1996. His research interests include wireless communications, spread spectrum technical, OFDM systems, and wireless sensor networks. He has published a large number of SCI Journal papers in the issues addressed physical layer for wireless communication systems. Moreover, he also majors in developing some applications of the IOT (Internet of Thing) techniques and Dr. Joy I.-Z. Chen owned some patents authorized by the Taiwan Intellectual Property Office (TIPO).

ISSN: 2582-1369 (online)

\footnotetext{
ISSN: $2582-1369($ online $)$
} 